\title{
Dissolution Enhancement of Rosuvastatin Calcium by Liquisolid Compact Technique
}

\author{
V. J. Kapure, V. V. Pande, and P. K. Deshmukh \\ Department of Pharmaceutics and Quality Assurance, H. R. Patel Institute of Pharmaceutical Education and Research, \\ Shirpur, Maharashtra 425405, India
}

Correspondence should be addressed to V. V. Pande; drvishalpande@gmail.com

Received 19 November 2012; Accepted 21 January 2013

Academic Editor: Nayanabhirama Udupa

Copyright (C) 2013 V. J. Kapure et al. This is an open access article distributed under the Creative Commons Attribution License, which permits unrestricted use, distribution, and reproduction in any medium, provided the original work is properly cited.

In present investigation liquisolid compact technique is investigated as a tool for enhanced dissolution of poorly water-soluble drug Rosuvastatin calcium (RVT). The model drug RVT, a HMG-Co A reductase inhibitor was formulated in form of directly compressed tablets and liquisolid compacts; and studied for in-vitro release characteristics at different dissolution conditions. In this technique, liquid medications of water insoluble drugs in non-volatile liquid vehicles can be converted into acceptably flowing and compressible powders. Formulated systems were assessed for precompression parameters like flow properties of liquisolid system, Fourior transform infra red spectra (FTIR) analysis, X-ray powder diffraction (XRPD), differential scanning calorimetry (DSC), and post compression parameters like content uniformity, weight variation, hardness and friability, disintegration test, wetting time, in vitro dissolution studies, effect of dissolution volume on drug release rate, and estimation of fraction of molecularly dispersed drug in liquid medication. As liquisolid compacts demonstrated significantly higher drug release rates, we lead to conclusion that it could be a promising strategy in improving the dissolution of poor water soluble drugs and formulating immediate release solid dosage forms.

\section{Introduction}

The poor dissolution rate of water-insoluble drugs is still a substantial problem confronting the pharmaceutical industry. A great number of new and, possibly, beneficial chemical entities do not reach the public merely because of their poor oral bioavailability due to inadequate dissolution $[1,2]$.

Over the years, various formulation techniques like the formation of water-soluble molecular complexes, drug micronization, solid dispersion, coprecipitation, lyophilisation, and microencapsulation are some of the major formulation tools which have been shown to eknhance the dissolution characteristics of water-insoluble drugs $[3,4]$. The liquisolid compacts are a new and promising addition towards such a novel aim. This technique is a compressible and freeflowing powdered form of liquid medication. Using this new formulation technique, a liquid medication may be converted into a dry-looking, nonadherent, free-flowing, and readily compressible powder by a simple blending with selected powder excipients referred to as the carrier (cellulose, starch, lactose, etc.) and coating (silica) materials [5-7]. Research shows that soft gelatin capsules contain a solubilised liquid drug with a higher and more consistent bioavailability than the conventional oral dosage forms, due to the active ingredient present in the solution. Liquisolid compact delivers active ingredients in form of soft gelatin capsule preparation which contains liquid; where non-volatile solvent is used to dissolve the solid drug. Therefore, the drug is held in the solution, even though it is in a tabletted or encapsulated dosage form. Consequently, drug dissolution properties will be improved [8-10].

The present work deals with formulation of liquisolid compact of RVT calcium, a poor water-soluble drug, and its evaluation for precompression and postcompression parameters. The effect of dissolution media and their volume on the in vitro drug release rate was also studied.

Theoretical Aspects. In the fundamental studies made by Spireas et al. $[8,9]$, flow and compression issue have been addressed with the use of the new mathematical model of 
liquisolid system which is based on the flowable ( $\phi$-value) and compressible ( $\psi$-number) liquid retention potentials of the constituents powder. The good flow and compression properties of the liquisolid system are encouraged by the large surface area and fine particle size.

According to the new theories, the carrier $(Q)$ and coating $(q)$ powder materials can retain only certain amount of liquid while maintaining acceptable flow and compression properties. Depending on the excipients ratio $(R)$ or the carrier: coating ratio of the powder system used, where

$$
R=\frac{Q}{q}
$$

$(R)$ represents the ratio between the weights of carrier $(Q)$ and coating $(q)$ materials present in the formulation. A freeflowing and compressible liquisolid system can be prepared only if the maximum liquid on the carrier material is not exceeded; such a characteristic amount of liquid is termed as the liquid load factor $\left(L_{f}\right)$ and defined as the ratio of the weight of the liquid medication $(W)$ over the weight of the carrier powder $(Q)$ in the system, which should be possessed by an acceptably flowing and compressible liquisolid system $[10,11]$. That is,

$$
L_{f}=\frac{W}{Q} .
$$

The flowable liquid retention potentials ( $\phi$-value) of powder excipients are considered to calculate the required ingredient quantities [8]. Hence, the powder excipients ratios $R$ and liquid load factors $L_{f}$ of the formulations are related as follows:

$$
L_{f}=\phi+\phi\left(\frac{1}{R}\right) .
$$

In order to calculate the required weights of the excipients used, first, from (3), $\phi$ and $\phi$ are constants; therefore, according to the ratio of the carrier/coat materials $(R)$, $L_{f}$ was calculated from the linear relationship of $L_{f}$ versus $1 / R$ [11]. Different weights of the liquid drug solution $(W)$ will be used depending on the liquid vehicle concentration; with the knowledge of $L_{f}$ and $W$, the appropriate quantities of carrier $\left(Q_{o}\right)$ and coating $\left(q_{o}\right)$ powder materials required to convert a given amount of liquid medication $(W)$ into an acceptably flowing and compressible liquisolid system could be calculated from (1) and (2).

\section{Experimental}

2.1. Materials. RVT was obtained as a gift sample from Cadila pharmaceuticals Ltd., Ahmadabad. Aerosil 200 was procured from Research Lab, Avicel PH 102 and propylene glycol were purchased from Loba Chemicals Mumbai, polyethylene glycol 400, Tween 80 , and sodium starch glycolate were purchased from Merck. All chemicals and reagent, and used were of analytical grade.

\subsection{Methodology}

2.2.1. Solubility Studies. The solubility of RVT in water and the three liquid vehicles which were used to prepare the liquisolid system, namely, propylene glycol, polyethylene glycol 400 , and Tween 80 , were studied by preparing a saturated solution of the drug in these solvents and their drug content was analysed spectrophotometrically. Also, rosuvstatin, was mixed with each of the previous solvents in $10 \mathrm{~mL}$ glass vial individually in such quantity so as to produce systems containing an excess of drug. The mixtures were sonicated for $24 \mathrm{~h}$ and then cooled down to $25^{\circ} \mathrm{C}$ under constant vibration. Accurately weighed quantities of the filtered supernatant solutions were further diluted with methanol after centrifugation and analyzed spectrophotometrically at $242.6 \mathrm{~nm}$ for their drug content. The results were extrapolated to determine the percent $\mathrm{w} / \mathrm{w}$ of RVT in its saturated solution with the solvent under investigation.

2.2.2. Preparation of Directly Compressed Tablets. A conventional formulation of RVT was directly compressed into cylindrical tablets, each containing $10 \mathrm{mg}$ drug. In addition, each tablet contained the following powder excipients: $140 \mathrm{mg}$ coarse granular microcrystalline cellulose (Avicel PH 102), $70 \mathrm{mg}$ lactose monohydrate, $10 \mathrm{mg}$ Aerosil 200, and $20 \mathrm{mg}$ sodium starch glycolate. Twenty tablets batches were mixed in a mortar for $10 \mathrm{~min}$ and compressed using a compression machine. Sufficient compression loads were applied in order to produce tablets of $3-5 \mathrm{~kg} / \mathrm{cm}^{2}$ hardness.

2.2.3. Preparation of Liquisolid Compacts. Several liquisolid systems of RVT were prepared in 20 tablet batches and compressed into cylindrical tablets of $10 \mathrm{mg}$ strength each, using compression machine and a target hardness of $3-5 \mathrm{~kg} / \mathrm{cm}^{2}$. All liquisolid formulations contained microcrystalline cellulose as the carrier powder and silica as the coating (covering) material at a fixed powder excipient ratio $(R)$ of 20. Propylene glycol was used as the liquid vehicle to prepare the liquid medications of different drug concentrations, ranging from 10 to $20 \% \mathrm{w} / \mathrm{w}$, included in the formulations LST-1 to LST-3. On the other hand, PEG 400 was used as the liquid vehicle in the formulations LST-4 to LST-6, and, similarly, Tween 80 was used as the liquid vehicle in the formulations LST-7 to LST9 with similar drug concentration. Depending on the liquid vehicle and drug concentration in the liquid medication used, different liquid load factors $\left(L_{f}\right)$ ranging from 0.195 to 0.235 $(\mathrm{w} / \mathrm{w})$ were employed in liquisolid preparations. Finally, a standard 5\% (w/w) of the disintegrant sodium starch glycolate was added to all liquisolid systems. Important formulation characteristics of RVT liquisolid compacts are shown in Table 1.

\subsection{Application of Mathematical Model for RVT Liquisolid System}

2.3.1. Determination of the Angle of Slide for Aerosil 200. The angle of slide for Aerosil 200 was measured by the following procedure. 
TABLE 1: Key formulation characteristics of prepared RVT liquisolid tablets.

\begin{tabular}{|c|c|c|c|c|c|c|c|}
\hline $\begin{array}{l}\text { Liquisolid } \\
\text { system }\end{array}$ & $\begin{array}{l}\text { Liquid } \\
\text { vehicle }\end{array}$ & $\begin{array}{l}\text { Drug concentration }(\% \mathrm{w} / \mathrm{w}) \\
\text { in liquid medication } \\
\left(C_{d}\right)^{*}\end{array}$ & $\begin{array}{c}\text { Excipient ratio } \\
(R)^{\dagger}\end{array}$ & $\begin{array}{l}\text { Avicel PH-102 } \\
(Q)^{\ddagger} \mathrm{mg}\end{array}$ & $\begin{array}{l}\text { Aerosil } 200 \\
(q)^{\wedge} \mathrm{mg}\end{array}$ & $\begin{array}{l}\text { Liquid load } \\
\text { factor } \\
\left(L_{f}\right)^{\#}\end{array}$ & $\begin{array}{c}\text { Molecular } \\
\text { fraction } \\
\left(F_{M}\right)^{+} \\
\end{array}$ \\
\hline LST-1 & PG & 10 & 20 & 166.6 & 8.33 & 0.235 & 1.074 \\
\hline LST-2 & PG & 15 & 20 & 249.9 & 12.49 & 0.235 & 0.716 \\
\hline LST-3 & PG & 20 & 20 & 333.2 & 16.66 & 0.235 & 0.537 \\
\hline LST-4 & PEG-400 & 10 & 20 & 227.1 & 11.35 & 0.168 & 0.452 \\
\hline LST-5 & PEG-400 & 15 & 20 & 343.0 & 17.15 & 0.168 & 0.301 \\
\hline LST-6 & PEG-400 & 20 & 20 & 454.3 & 22.71 & 0.168 & 0.226 \\
\hline LST-7 & Tween-80 & 10 & 20 & 287.7 & 14.38 & 0.195 & 0.348 \\
\hline LST-8 & Tween-80 & 15 & 20 & 431.5 & 21.57 & 0.195 & 0.232 \\
\hline LST-9 & Tween-80 & 20 & 20 & 575.3 & 28.76 & 0.195 & 0.174 \\
\hline
\end{tabular}

* An appropriate amount of liquid medication containing $10 \mathrm{mg}$ of drug was incorporated in each tablet.

${ }^{\dagger}$ Excipient ration $(R)$ equal to 20 in each formulation.

${ }^{\ddagger}$ Amount of carrier $(Q)$ material.

$\wedge$ Amount of coating $(q)$ material.

${ }^{\#}$ Liquid load factor is defined as $L_{f}=W / Q$.

${ }^{+}$The fraction $\left(F_{M}\right)$ of molecularly dispersed drug was calculated on the basis of (7).

Ten grams of Aerosil 200 were weighed accurately and placed at one end of a metal plate with a polished surface. This end was raised gradually until the plate made an angle with the horizontal surface at which the powder particles starts to slide. An angle of slide corresponds to $33^{\circ}$ shown optimal flow properties [12].

2.3.2. Determination of Flowable Liquid Retention Potential for Aerosil ( $\phi$-Value). An increasing amount of liquid vehicle (PG, PEG400, and Tween 80) was added to the $10 \mathrm{~g}$ of Aerosil 200 powder and mixed well. The Aerosil adsorbed the liquid vehicle resulting into change in its flow properties. The angle of slide for aerosil was redetermined as stated earlier. The corresponding $\phi$-value was calculated from the following equation:

$$
\phi \text {-value }=\frac{\text { weight of liquid }}{\text { weight of solid }} .
$$

The $\phi$-values were plotted graphically against the corresponding angle of slide which represented the flowable liquid retention potential of Aerosil.

According to Tayel et al. in propylene glycol, the $\phi$-value is 0.16 for Avicel PH 102 and 1.5 for Aerosil 200. And for PEG 400 , the $\phi$-value is 0.005 for Avicel PH 102 and 3.26 for Aerosil 200 , thus showing no need to determine it practically. The liquid load factor for PG and PEG 400 liquisolid system was calculated from flowable liquid retention potential using $R$ value (excipient ratio) of 20, which is as follows [12]:

$$
\begin{gathered}
L_{f}=\phi+\phi\left(\frac{1}{R}\right), \\
L_{f}=0.16+1.5\left(\frac{1}{20}\right)=0.235, \\
L_{f}=0.005+1.5\left(\frac{1}{20}\right)=0.168 .
\end{gathered}
$$

\subsection{Precompression Studies of Liquisolid System}

2.4.1. Flow Properties of Liquisolid System. The flow properties of liquisolid system were estimated by determining the angle of repose, Carr's index, and Hausner's ratio. The angle of repose was measured by the fixed funnel method. The bulk density and tapped densities were determined for the calculation of Hausner's ratio and Carr's index [13, 14].

2.4.2. Infrared Spectra Analysis. IR spectra of the liquisolid system were recorded by the $\mathrm{KBr}$ pellet method using the Fourier transform infrared spectrophotometer. A baseline correction was made using dried potassium bromide, and then the spectrum of pure RVT and liquisolid system was obtained.

2.4.3. X-Ray Powder Diffraction (XRPD). X-ray diffractograms of pure RVT and liquisolid formulation were obtained using Philips Analytical XRD instrument. The scanning range was from 5 to $80^{\circ}$ at 2 theta scale.

2.4.4. Differential Scanning Calorimetry (DSC). Thermograms of the pure RVT and liquisolid system were recorded on a DSC. The thermal behaviour of the samples was investigated at a scanning rate of $10^{\circ} \mathrm{C} / \mathrm{min}$, covering a temperature range of $40-2600^{\circ} \mathrm{C}$.

\subsection{Post Compression Studies of Liquisolid Compacts}

2.5.1. Content Uniformity. In uniformity of drug content, 10 tablets from each batch were taken randomly to examine its content uniformity. Each tablet was weighed and crushed individually. The crushed tablet powders were dissolved in methanol. The solution was filtered using Whatman filter paper. The drug content was measured using UV spectrophotometer at $242.6 \mathrm{~nm}$. According to the British Pharmacopoeia 
the percentages drug content of individual were calculated against the average drug content [15].

2.5.2. Weight Variation. The weight variation test was performed on 20 tablets of liquisolid compacts as per British Pharmacopoeia [15].

2.5.3. Hardness and Friability. The hardness of formulated liquisolid tablets was determined by using Pfizer hardness tester, and the mean hardness of the three liquisolid tablets was determined. The friability of the prepared liquisolid tablets was measured using Roche type apparatus, and the drum was rotated for $4 \mathrm{~min}$ at $25 \mathrm{rpm}$. The losses of the mass of 10 tablets were determined, and by using (6), the percentage of friability was calculated [16] as follow:

$$
\% \text { Friability }=\left(\frac{\text { loss of mass }}{\text { Initial mass }}\right) \times 100 \text {. }
$$

2.5.4. Disintegration Test. The disintegration test was performed at $37 \pm 1^{\circ} \mathrm{C}$ in distilled water for six tablets from each formulation using the tablet disintegration unit. The tablets were considered completely disintegrated as no residue remains on the screen. Generally, ideal tablet hardness should be produced without applying excessive compression force where rapid tablet disintegration and drug dissolution are maintained at the same time [17].

2.5.5. Wetting Time. Wetting is the important step for disintegration process to take place. A piece of tissue paper folded double was placed in a petri dish containing $6 \mathrm{~mL}$ of water. The tablet was placed on the paper, and the time for complete wetting of the tablet was measured in seconds. Wetting time corresponds to the time taken for the tablet to disintegrate when kept motionless on the tongue [18].

2.5.6. In Vitro Dissolution Studies and Effect of Dissolution Volume on Drug Release Rate. In vitro dissolution profile from liquisolid compacts and directly compressed tablets was obtained using dissolution test apparatus USP-II. The dissolution studies were carried out in $900 \mathrm{~mL}, 450 \mathrm{~mL}$, and $300 \mathrm{~mL}$ of $1.2 \mathrm{pH}$ buffer and distilled water as the dissolution medium at $37^{\circ} \mathrm{C} \pm 1^{\circ} \mathrm{C}$ and $50 \mathrm{rpm}$. Then, $5 \mathrm{~mL}$ samples were collected for up to $60 \mathrm{~min}$ at $5 \mathrm{~min}$ intervals up to $30 \mathrm{~min}$ and $15 \mathrm{~min}$ intervals from 30 to $60 \mathrm{~min}$. The dissolution medium was replaced with $5 \mathrm{~mL}$ fresh dissolution fluid to maintain the sink condition. The withdrawn samples were filtered and analysed spectrophotometrically at $242.6 \mathrm{~nm}$.

2.5.7. Estimation of Fraction of Molecularly Dispersed Drug in Liquid Medication. The fraction $\left(F_{M}\right)$ of the dissolved or molecularly dispersed drug in the liquid medication is the ratio of the drug's saturation solubility $\left(C_{L}\right)$ in the liquid
TABLE 2: Solubility of RVT in different solvents.

\begin{tabular}{lc}
\hline Solvent & $\begin{array}{c}\text { Solubility }(\% \mathrm{w} / \mathrm{w}) \\
\left(C_{L}\right)\end{array}$ \\
\hline Distilled water & 0.0125 \\
Propylene glycol & 10.7481 \\
Polyethylene glycol 400 & 4.5286 \\
Polysorbate 80 (Tween 80$)$ & 3.4814 \\
\hline
\end{tabular}

$C_{L}$ : the saturation solubility of drug in non-volatile liquid vehicle.

vehicle (Table 2) over the drug concentration $\left(C_{d}\right)$ in the liquid medication:

$$
F_{M}=\frac{C_{L}}{C_{d}}
$$

where $F_{M}=1$ when $C_{L} / C_{d}>1$.

The value of fraction of the molecularly dispersed drug $F_{M}$ (Table 1$)$ in any system cannot exceed unity.

\section{Result and Discussion}

3.1. Solubility Study. RVT was selected as the model drug for these studies, since it is a water-insoluble drug and thus an ideal candidate for testing the potential of rapid release liquisolid compact. In addition, it can be easily determined in solution using spectrophotometric principles and procedures. Beer's law was obeyed by all the standard curves of our RVT solution which were linear in the concentration range, that is, from 1 to $14 \mu \mathrm{g} / \mathrm{mL}$. The solubility of RVT in water, propylene glycol, polyethylene glycol 400 , and polysorbate 80 (Tween 80 ) determined in these studies are shown in Table 2.

\subsection{Precompression Studies of the Liquisolid System}

3.2.1. Flow Properties of the RVT Liquisolid System. The flow properties of the liquisolid powder system are influenced by physical, mechanical, and environmental factors. Therefore, different flow properties were employed. As the angle of repose $(\theta)$ is a characteristic of the internal friction or cohesion of the particles, the value of angle of repose will be high if the powder is cohesive and low if the powder is noncohesive. LS-1 shows a good flow property with $\theta$ value of 29.59 and is considered as a liquisolid system with acceptable flowability. Carr's index up to 16 was considered acceptable as a flow property. Hausner's ratio was related to the interparticle friction; powders with a low interparticle friction had a ratio of approximately 1.25 indicating a good flow. The LS-1 system with Carr's index of 14.63 and Hausner's ratio of 1.17 was considered for further study, and results of all batches of RVT liquisolid compacts are shown in Table 3.

3.2.2. IR Spectra Analysis. Samples of Aerosil 200, Avicel PH102 , pure RVT, and liquisolid system (LS-1) were subjected to FT-IR spectroscopic analysis, and their spectra at 500$4000 \mathrm{~cm}^{-1}$ are shown in Figure 1. Characteristics peaks of aromatic $\mathrm{N}-\mathrm{H}$ stretching and $\mathrm{C}=\mathrm{O}$ stretching at $3364.57 \mathrm{~cm}^{-1}$ and $1556.55 \mathrm{~cm}^{-1}$ appeared, respectively. A reduction in 


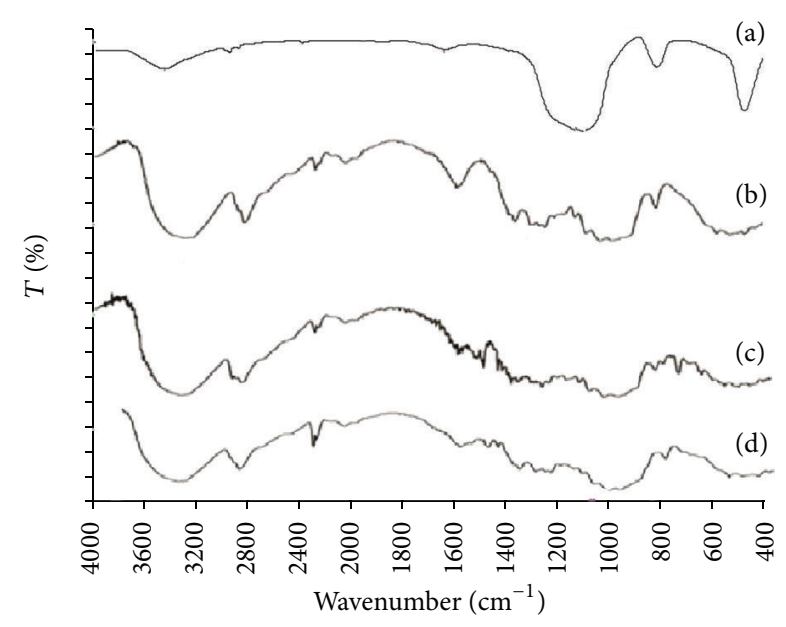

Figure 1: FT-IR spectra of (a) Aerosil 200, (b) Avicel PH 102, (c) pure RVT, and (d) liquisolid system (LS-1).

the intensity of the characteristics absorption bands of RVT was observed in the liquisolid formulation, which might be attributed to the hydrogen bonding interaction between the carboxylic group of RVT and the hydroxyl group of the liquid vehicles; this resulted in drug dissolution enhancement.

3.2.3. X-Ray Powder Diffraction (XRPD). Polymorphic changes in the drug are important since they might affect the dissolution rate and in-line bioavailability. Hence, it was necessary to study the polymorphic changes of RVT in liquisolid compacts. Figure 2 shows XRPD of pure RVT (a) and liquisolid system (b). X-ray diffraction pattern in Figure 2 demonstrated that pure RVT was clearly in crystalline state as it showed sharp distinct peaks at $2 \theta$ diffraction angles of $15.5^{\circ}, 22.6^{\circ}, 31.7^{\circ}, 34.5^{\circ}$, and $45.5^{\circ}$. XRPD pattern of formed liquisolid showed absence of sharp peaks. Such an absence of RVT constructive reflection (specific sharp peak) in the liquisolid X-ray diffractogram indicates that RVT has almost entirely converted from crystalline to amorphous or solubilised form; such lack of crystallinity in the liquisolid system was understood to be a result of RVT solubilisation in the liquid vehicle that was absorbed into and adsorbed onto the carrier and coating material.

3.2.4. Differential Scanning Calorimetry (DSC). The possible interaction between a drug entity and excipient in liquisolid compact was determined by DSC. Figure 3 shows thermal behaviour of the pure component (a) together with the thermal behaviour of the formulated liquisolid system (b). Pure RVT shows two characteristic peaks at $80^{\circ} \mathrm{C}$ and $164^{\circ} \mathrm{C}$ this is because of the polymorphic form of RVT, that is, form " $\mathrm{S}$ ", and it is a primary indication for crystalline nature of pure drug. Furthermore, thermal behaviour of liquisolid system (LS-1) shows the shifting of peak at $143^{\circ} \mathrm{C}$. It indicates that crystalline nature of drug gets completely converted into amorphous form due to which there is a significant change in endothermic peak of formed liquisolid system.

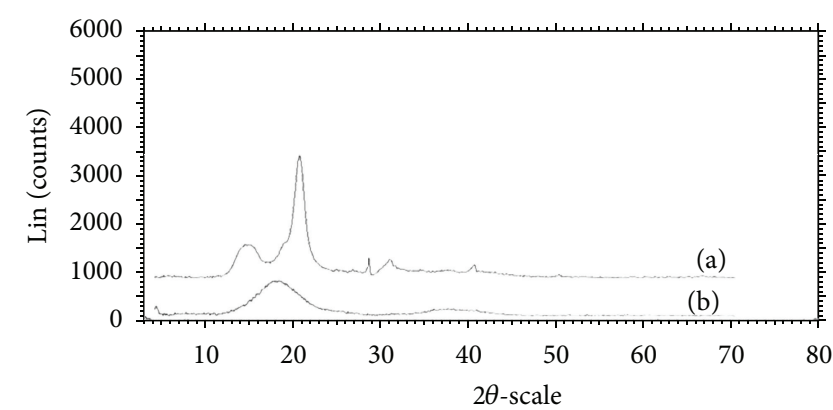

FIgURE 2: X-ray diffractogram of (a) pure drug RVT and (b) liquisolid system LS-1.

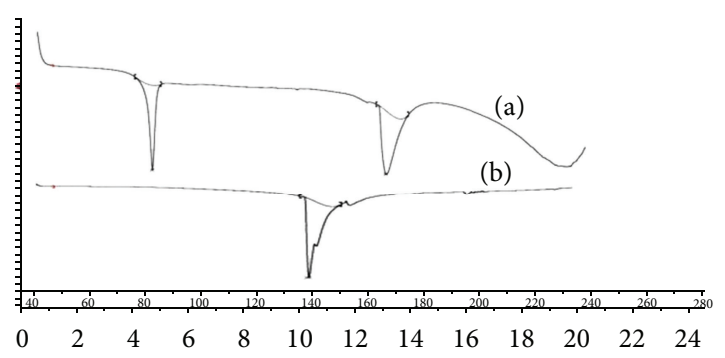

FIGURE 3: DSC thermograms of (a) pure RVT and (b) liquisolid system LS-1.

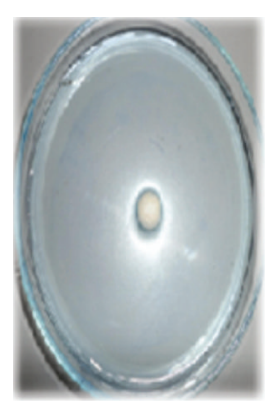

After $5 \mathrm{sec}$

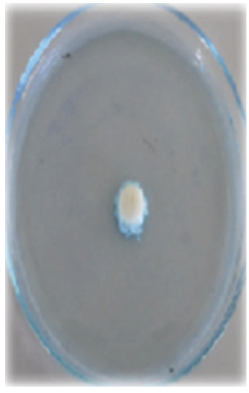

After $10 \mathrm{sec}$

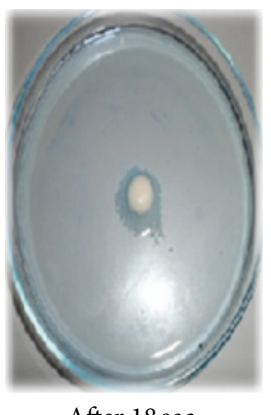

After $18 \mathrm{sec}$
FIGURE 4: The wetting time of liquisolid system (LS-1) in seconds.

\subsection{Post Compression Studies of Liquisolid Compacts}

3.3.1. Content Uniformity. All the liquisolid and conventional tablets complied with the British Pharmacopoeia (BP) weight uniformity test; also, all the tablets had met content uniformity criteria, as per BP, in which each individual content was between $85 \%$ and $115 \%$ of the average content.

\subsubsection{Friability, Hardness, Weight Variation, Disintegration} Test, and Fraction of Molecularly Dispersed Drug $\left(F_{M}\right)$. All the RVT liquisolid tablets exhibit the acceptable friability. The percentage did not exceed $1 \%$ of the tablet weight, and no tablet was broken or deformed. Since all the prepared formulae met the standard friability criteria, they are expected to show acceptable toughness and withstand 
TABLE 3: Flow properties of RVT liquisolid systems.

\begin{tabular}{|c|c|c|c|c|c|}
\hline Liquisolid system & Angle of repose & Bulk density $\left(\mathrm{gm} / \mathrm{cm}^{3}\right)$ & Tapped density $\left(\mathrm{gm} / \mathrm{cm}^{3}\right)$ & Carr's index & Hausner's ratio \\
\hline LS-1 & $29.59 \pm 0.51$ & $0.24 \pm 0.01$ & $0.28 \pm 0.02$ & $14.63 \pm 0.21$ & $1.17 \pm 0.01$ \\
\hline LS-2 & $30.28 \pm 0.24$ & $0.23 \pm 0.02$ & $0.28 \pm 0.02$ & $16.69 \pm 0.32$ & $1.20 \pm 0.01$ \\
\hline LS-3 & $31.50 \pm 0.32$ & $0.23 \pm 0.02$ & $0.27 \pm 0.01$ & $15.30 \pm 0.15$ & $1.18 \pm 0.04$ \\
\hline LS-4 & $30.45 \pm 0.45$ & $0.28 \pm 0.04$ & $0.33 \pm 0.04$ & $15.69 \pm 0.53$ & $1.18 \pm 0.01$ \\
\hline LS-5 & $30.83 \pm 0.22$ & $0.23 \pm 0.01$ & $0.28 \pm 0.08$ & $17.87 \pm 0.12$ & $1.21 \pm 0.02$ \\
\hline LS-6 & $33.86 \pm 0.43$ & $0.25 \pm 0.03$ & $0.31 \pm 0.01$ & $19.00 \pm 0.14$ & $1.23 \pm 0.01$ \\
\hline LS-7 & $32.82 \pm 0.55$ & $0.27 \pm 0.02$ & $0.32 \pm 0.03$ & $16.25 \pm 0.42$ & $1.19 \pm 0.05$ \\
\hline LS-8 & $33.86 \pm 0.24$ & $0.27 \pm 0.02$ & $0.33 \pm 0.15$ & $18.05 \pm 0.53$ & $1.22 \pm 0.04$ \\
\hline LS-9 & $36.72 \pm 0.25$ & $0.25 \pm 0.04$ & $0.31 \pm 0.05$ & $20.25 \pm 0.12$ & $1.25 \pm 0.01$ \\
\hline DCT & $37.77 \pm 0.43$ & $0.28 \pm 0.01$ & $0.34 \pm 0.02$ & $18.32 \pm 0.24$ & $1.22 \pm 0.01$ \\
\hline
\end{tabular}

TABLE 4: Physical parameter of liquisolid tablet.

\begin{tabular}{lcccccc}
\hline $\begin{array}{l}\text { Liquisolid } \\
\text { system }\end{array}$ & $\begin{array}{c}\text { Hardness } \\
\left(\mathrm{kg} / \mathrm{cm}^{2}\right)\end{array}$ & $\begin{array}{c}\text { Thickness } \\
(\mathrm{mm})\end{array}$ & $\begin{array}{c}\text { Disintegration time } \\
(\mathrm{sec})\end{array}$ & $\begin{array}{c}\text { Friability } \\
(\%)\end{array}$ & $\begin{array}{c}\text { Weight variation } \\
(\mathrm{mg})\end{array}$ & $\begin{array}{c}\text { Content uniformity } \\
(\%)\end{array}$ \\
\hline LS-1 & $3.82 \pm 0.28$ & $3.4 \pm 0.02$ & $14 \pm 0.28$ & 0.21 & $241.8 \pm 1.1$ & $99.25 \pm 0.24$ \\
LS-2 & $4.20 \pm 0.42$ & $3.0 \pm 0.01$ & $16 \pm 0.51$ & 0.30 & $253.4 \pm 0.8$ & $98.25 \pm 0.20$ \\
LS-3 & $4.62 \pm 0.31$ & $2.9 \pm 0.01$ & $18 \pm 0.50$ & 0.45 & $341.5 \pm 1.8$ & $98.00 \pm 0.51$ \\
LS-4 & $3.91 \pm 0.14$ & $3.4 \pm 0.02$ & $17 \pm 0.32$ & 0.33 & $272.1 \pm 1.2$ & $96.51 \pm 0.44$ \\
LS-5 & $4.42 \pm 0.31$ & $3.2 \pm 0.04$ & $22 \pm 0.40$ & 0.45 & $368.4 \pm 1.6$ & $97.26 \pm 0.23$ \\
LS-6 & $4.71 \pm 0.15$ & $3.0 \pm 0.02$ & $26 \pm 0.14$ & 0.53 & $483.1 \pm 0.4$ & $98.60 \pm 0.45$ \\
LS-7 & $4.15 \pm 0.18$ & $3.7 \pm 0.01$ & $19 \pm 0.21$ & 0.29 & $332.5 \pm 1.0$ & $98.21 \pm 0.50$ \\
LS-8 & $4.54 \pm 0.35$ & $3.4 \pm 0.03$ & $24 \pm 0.53$ & 0.37 & $492.2 \pm 0.7$ & $95.27 \pm 0.15$ \\
LS-9 & $4.62 \pm 0.23$ & $2.9 \pm 0.01$ & $28 \pm 0.42$ & 0.34 & $638.1 \pm 1.5$ & $97.51 \pm 0.25$ \\
DCT & $4.80 \pm 0.22$ & $3.5 \pm 0.01$ & $44 \pm 0.15$ & 0.48 & $248.4 \pm 0.5$ & $99.00 \pm 0.42$ \\
\hline
\end{tabular}

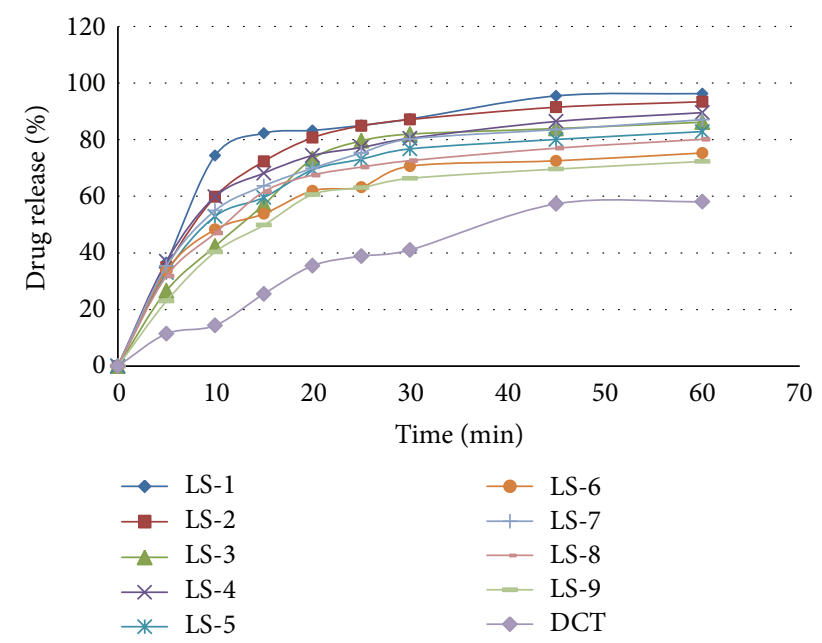

FIGURE 5: Dissolution profile of RVT liquisolid compacts and direct compressed tablet in $1.2 \mathrm{pH}$.

abrasion during handling. All the prepared batches had hardness in range $3-5 \mathrm{~kg} / \mathrm{cm}^{2}$. Generally, the ideal tablet hardness should be produced without applying excessive compression force where rapid tablet disintegration and drug dissolution are maintained at the same time [16]. Also, the

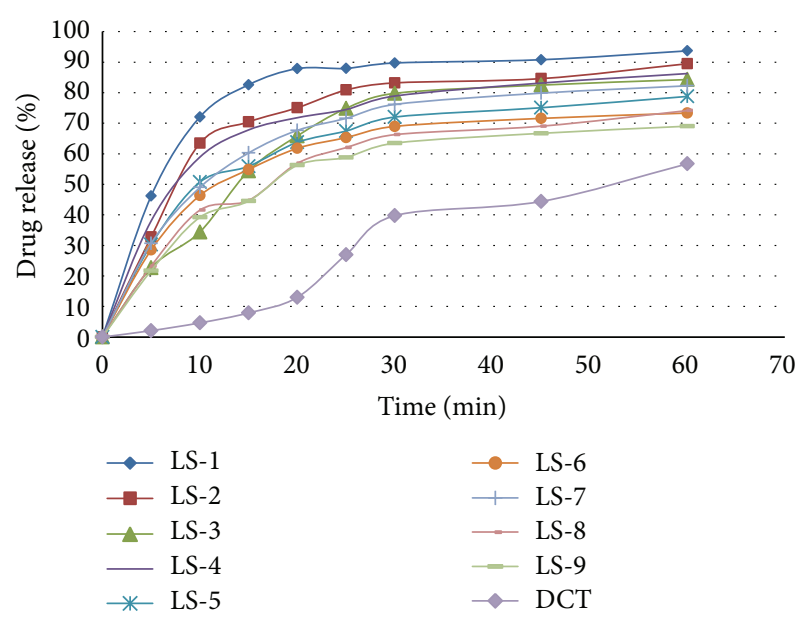

FIGURE 6: Dissolution profile of RVT liquisolid compacts and directly compressed tablet in distilled water.

batches passed the USP weight variation test. All the prepared batches had a disintegration time less than $1 \mathrm{~min}$. The batches prepared with increasing drug concentration exhibited an increasing disintegration time. Based on (6), $F_{M}$ value of each liquisolid preparation was calculated. No liquid vehicle 

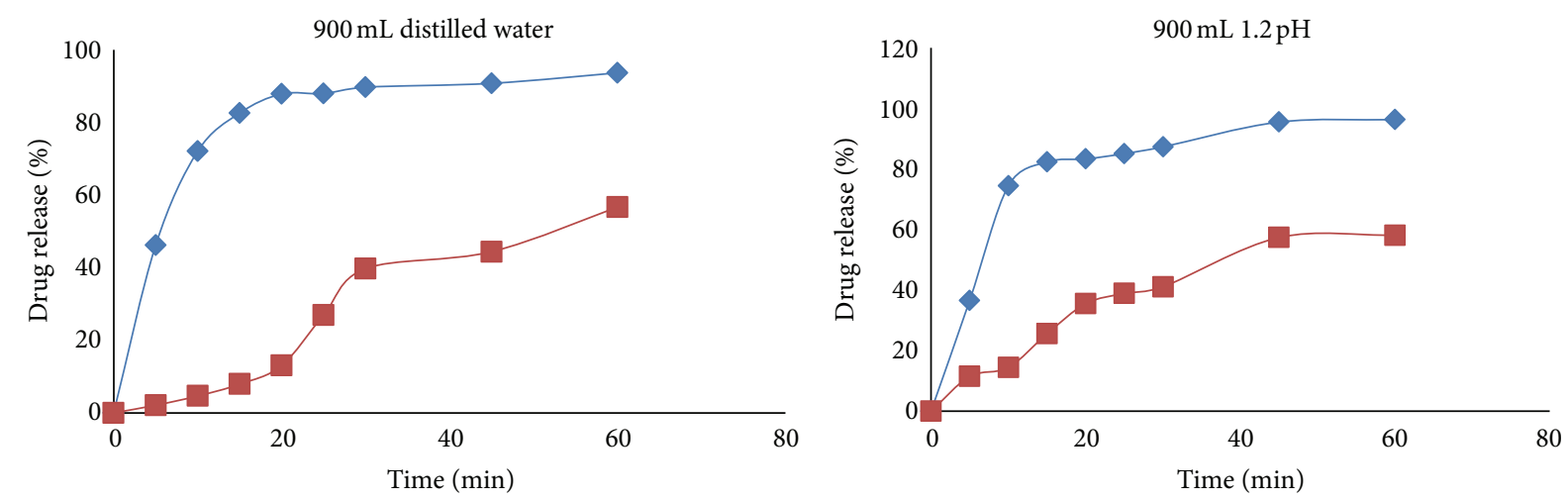

(a)
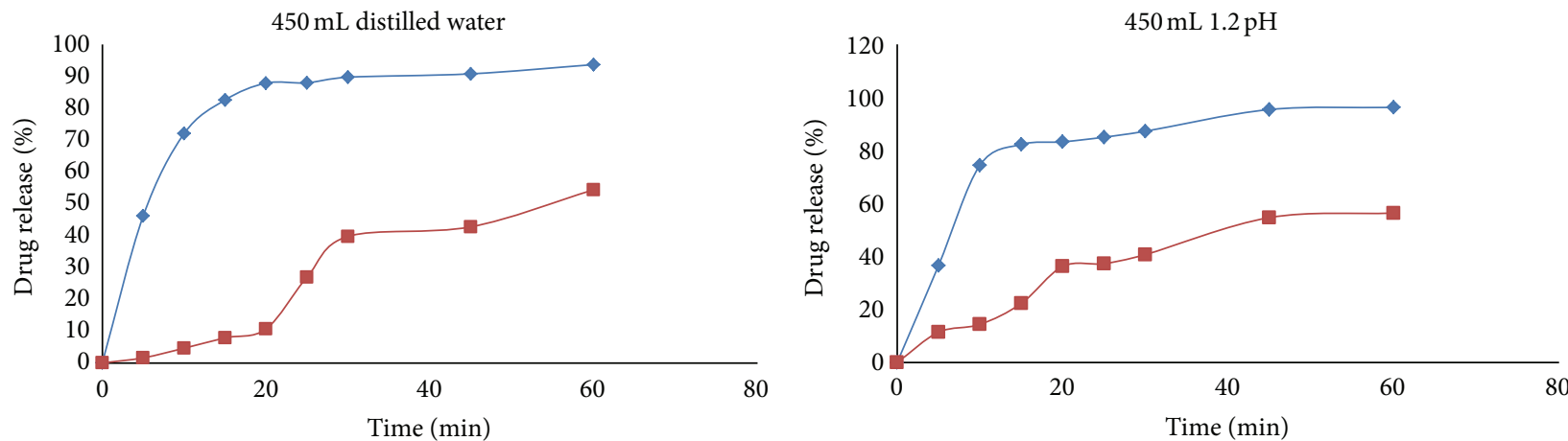

(b)
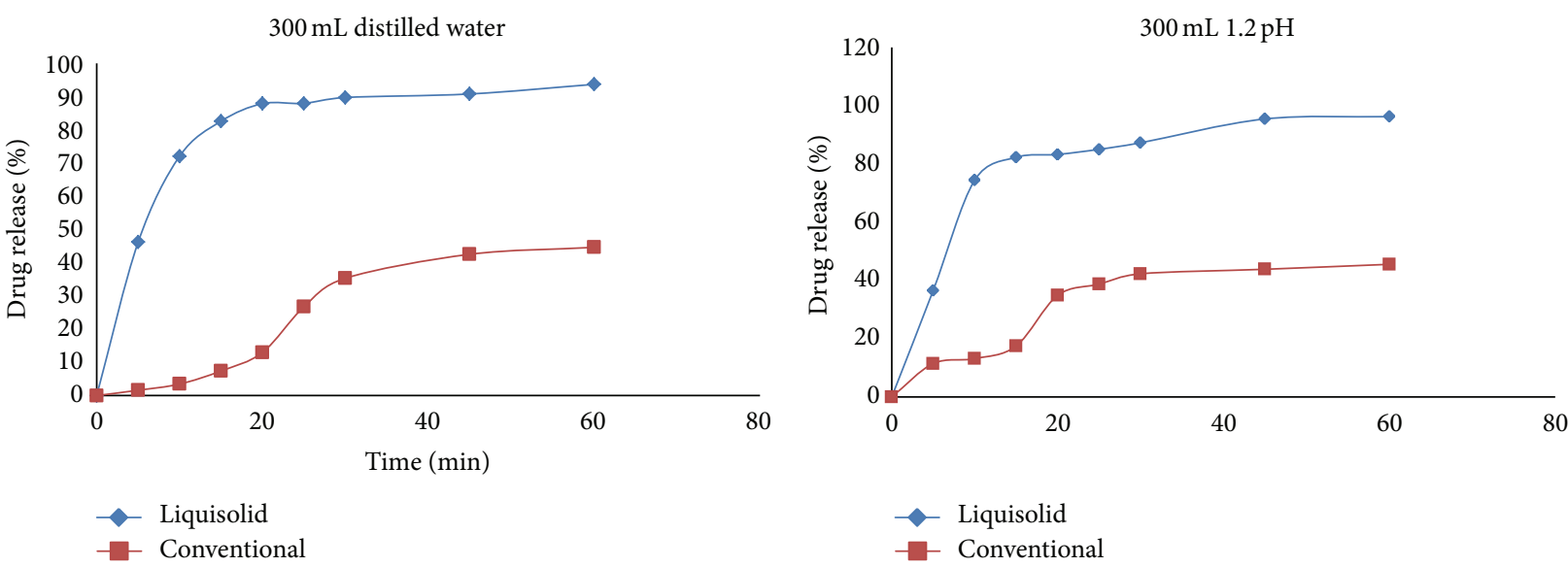

(c)

FIGURE 7: Comparison of dissolution profile displayed by the liquisolid compact (LS-1) and conventional tablet in $900 \mathrm{~mL}, 450 \mathrm{~mL}$ and $300 \mathrm{~mL}$ volume of distilled water and $1.2 \mathrm{pH}$.

is involved in the case of directly compressed tablets which contain plain micronized RVT powder, and their $F_{M}$ value was taken as 0 . The result of friability, hardness, weight variation, disintegration time, and fraction of molecularly dispersed drug $\left(F_{M}\right)$ for all the batches of RVT liquisolid compacts are shown in Table 4.

3.3.3. Wetting Time. Wetting time is closely related to the inner structure of the tablets and to the hydrophilicity of the excipient. A linear relationship exists between wetting time and disintegration time. The wetting time of liquisolid system (LS-1) was found to be 18-20 sec as compared to the conventional tablet which showed wetting time as $35-38 \mathrm{sec}$. The wetting time for liquisolid system (LS-1) was shown in Figure 4.

3.3.4. In Vitro Dissolution Studies and Effect of Dissolution Volume on Drug Release Rate. Figures 5 and 6 showed the 


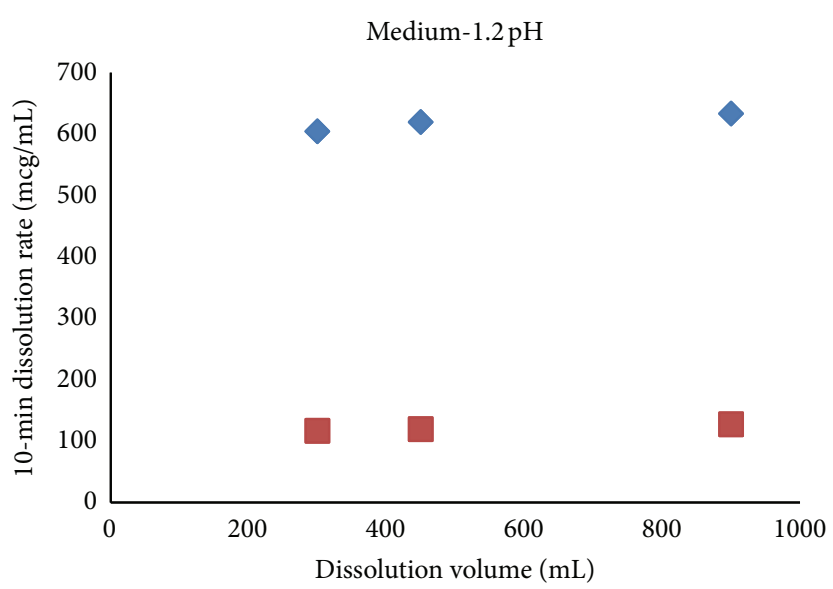

(a)

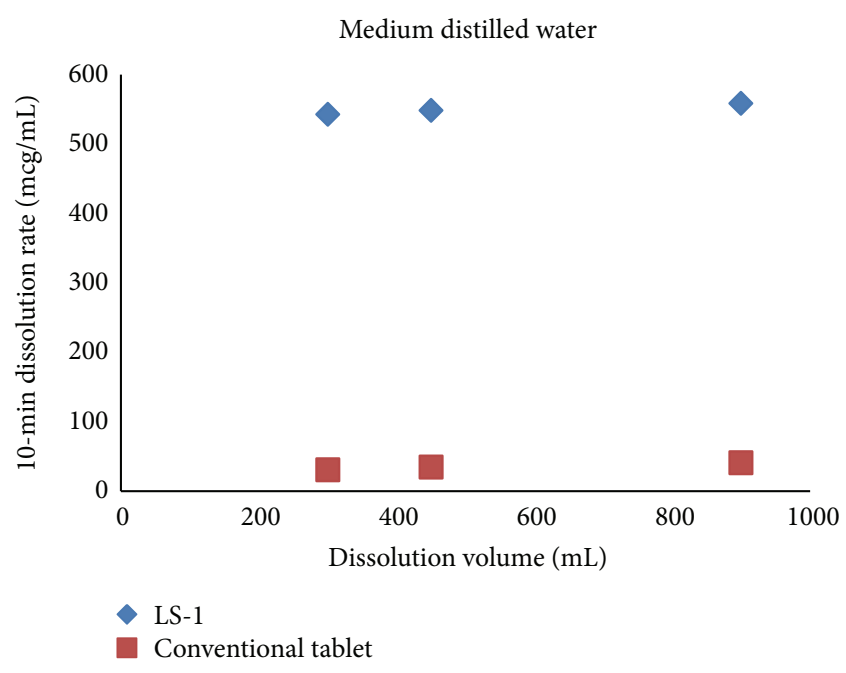

(b)

FIGURE 8: Effect of the dissolution volume on the initial dissolution rate of liquisolid compact (LS-1) exhibited by the liquisolid compacts and directly compressed tablets using different dissolution media.

dissolution profile of liquisolid compact of LS-1 to LS-9 with direct compressible tablet in $1.2 \mathrm{pH}$ and distilled water. Liquisolid compact LS-1 produced a higher dissolution in $1.2 \mathrm{pH}$ and distilled water in comparison with conventional tablet.

Figure 7 shows the drug dissolution profiles from the liquisolid compacts (LS-1) and the directly compressed tablets (DCT-1) of micronized RVT calcium. When $900 \mathrm{~mL}$ (per vessel) of distilled water or $1.2 \mathrm{pH}$ solution was used as the dissolving medium, liquisolid tablets displayed slightly better in vitro release characteristics than those of their directly compressed counterparts.

However, when smaller volumes, such as 450 and $300 \mathrm{~mL}$, of dissolution media's were used, the liquisolid tablets demonstrated significantly improved drug dissolution properties.

It seems that the drug dissolution rate of liquisolid compacts is significantly faster than that of the plain tablets, and it is independent of the volume of the dissolving liquid used. Furthermore, it is apparent that decreasing dissolution volumes result in a proportional decrease of the in vitro drug release rates displayed by the directly compressed tablets. According to the "diffusion layer model" dissolution theories, the dissolution rate of a drug is directly proportional to its concentration gradient $\left(\Delta C=C_{s}-C\right)$ in the stagnant diffusion layer formed by the dissolving liquid around the drug particles. $C_{s}$ is the saturation solubility of the drug in the dissolution medium, and, thus, it is a constant characteristic property related to the drug and dissolving liquid involved. On the other hand, $C$, the drug concentration in the bulk of the dissolving medium, increases with decreasing volumes of dissolution fluid used. Therefore, the $\Delta C$ values existing in the three different dissolution volumes of our tests decrease with decreasing volumes of dissolution medium. Consequently such $\Delta C$ reduction is directly related to the decreased drug dissolution rates of the conventional tablets with the decreasing volumes of both the dissolution media used (Figure 8).

\section{Conclusion}

The overall objective of present study was to enhance dissolution of poorly water-soluble RVT by liquisolid compact technique. The liquisolid tablets formulated with the propylene glycol at drug concentration of $10 \% \mathrm{w} / \mathrm{w}$ is the best formulation among all the batches of liquisolid tablets prepared, in terms of faster disintegration time, superior dissolution profile, and acceptable tablet properties. Propylene glycol was found to be a promising liquid vehicle in formulating liquisolid formulations of RVT calcium. The liquid vehicle plays a contributing role in improving the dissolution profiles of a poor water-soluble drug in the liquisolid formulations, besides choosing a suitable liquid vehicle according to its viscosity and HLB value. The key step in formulating a successful liquisolid tablet is the determination of the optimal flowable liquid retention potential ( $\phi$-value).

\section{Conflict of Interests}

The authors report no conflict of interests.

\section{Acknowledgment}

The authors are grateful to the management of HRPIPER, Shirpur, for providing necessary facilities to carry out research work.

\section{References}

[1] R. Löbenberg and G. L. Amidon, "Modern bioavailability, bioequivalence and biopharmaceutics classification system. New scientific approaches to international regulatory standards," European Journal of Pharmaceutics and Biopharmaceutics, vol. 50, no. 1, pp. 3-12, 2000. 
[2] M. N. Martinez and G. L. Amidon, "A mechanistic approach to understanding the factors affecting drug absorption: a review of fundamentals," Journal of Clinical Pharmacology, vol. 42, no. 6, pp. 620-643, 2002.

[3] G. Chawla and A. K. Bansal, "A comparative assessment of solubility advantage from glassy and crystalline forms of a water-insoluble drug," European Journal of Pharmaceutical Sciences, vol. 32, no. 1, pp. 45-57, 2007.

[4] G. R. Lloyd, D. Q. M. Craig, and A. Smith, "A calorimetric investigation into the interaction between paracetamol and polyethlene glycol 4000 in physical mixes and solid dispersions," European Journal of Pharmaceutics and Biopharmaceutics, vol. 48, no. 1, pp. 59-65, 1999.

[5] K. Rajesh, R. Rajalakshmi, J. Umamaheswari, and C. Ashok, "Liquisolid technique a novel approach to enhance solubility and bioavailability," International Journal of Biopharmaceutics, vol. 2, pp. 8-13, 2011.

[6] S. Mallick, S. K. Pradhan, M. Chandran, M. Acharya, T. Digdarsini, and R. Mohapatra, "Study of particle rearrangement, compression behaviour and dissolution properties after melt dispersion of ibuprofen, Avicel and Aerosil," Results in Pharma Sciences, vol. 1, no. 1, pp. 1-10, 2011.

[7] B. Jayanthi, R. Manavalan, and P. K. Manna, "Preparation and evaluation of aceclofenac ethyl cellulose micro particles using aerosil as dispersing carrier," Research Journal of Pharmaceutical, Biological and Chemical Sciences, vol. 3, no. 1, pp. 67-77, 2012.

[8] S. Spireas and S. Bolton, "Liquisolid system and methods of preparing Same," U.S. Patent no. 5,968,550, October 1999.

[9] S. Spireas and S. Sadu, "Enhancement of prednisolone dissolution properties using liquisolid compacts," International Journal of Pharmaceutics, vol. 166, no. 2, pp. 177-188, 1998.

[10] N. Tiong and A. A. Elkordy, "Effects of liquisolid formulations on dissolution of naproxen," European Journal of Pharmaceutics and Biopharmaceutics, vol. 73, no. 3, pp. 373-384, 2009.

[11] R. H. Fahmy and M. A. Kassem, "Enhancement of famotidine dissolution rate through liquisolid tablets formulation: In vitro and in vivo evaluation," European Journal of Pharmaceutics and Biopharmaceutics, vol. 69, no. 3, pp. 993-1003, 2008.

[12] S. A. Tayel, I. I. Soliman, and D. Louis, "Improvement of dissolution properties of Carbamazepine through application of the liquisolid tablet technique," European Journal of Pharmaceutics and Biopharmaceutics, vol. 69, no. 1, pp. 342-347, 2008.

[13] J. Staniforth, "Powder flow," in Pharmaceutics: The Science of Dosage Form Design, M. E. Aulton, Ed., pp. 197-210, Churchill Livingstone, Longman group, Edinburg, UK, 2nd edition, 2002.

[14] R. Shangraw, "Compressed tablets by direct compression," in Pharmaceutical Dosage Forms: Tablets, H. A. Lieberman, L. Lachman, and J. B. Schwartz, Eds., pp. 195-245, Marcel Dekker, New York, NY, USA, 2nd edition, 1989.

[15] British Pharmacopoeia, vol. 4, 2008.

[16] A. Martin, P. Bustamante, and A. Chun, "Micromeritics," in Martin's Physical Pharmacy and Pharmaceutical Sciences, P. J. Sinko, Ed., pp. 533-560, Lippincott Williams \& Wilkins, Philadelphia, Pa, USA, 5th edition, 2006.

[17] R. Shangraw, "Compressed tablets by direct compression," in Pharmaceutical Dosage Forms: Tablets, H. A. Lieberman, L. Lachman, and J. B. Schwartz, Eds., pp. 195-246, Marcel Dekker, New York, NY, USA, 2nd edition, 1989.

[18] M. Sivakranth, A. S. Althaf, and S. Rajasekhar, "Formulation and evaluation of oral fast dissolving tablets of sildenafil citrarte," International Journal of Pharmacy and Pharmaceutical Sciences, vol. 3, supplement 2, pp. 112-121, 2011. 

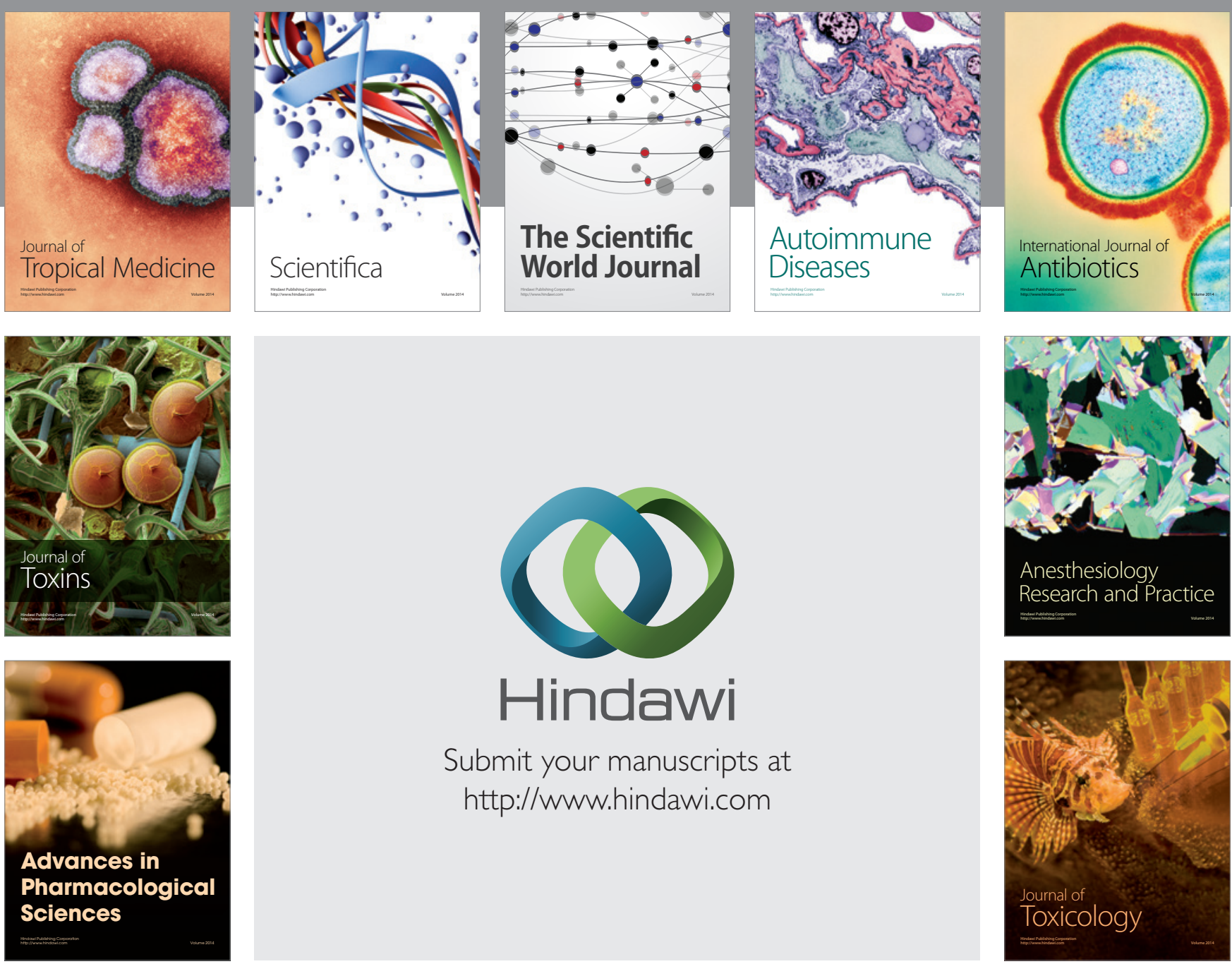

\section{Hindawi}

Submit your manuscripts at

http://www.hindawi.com
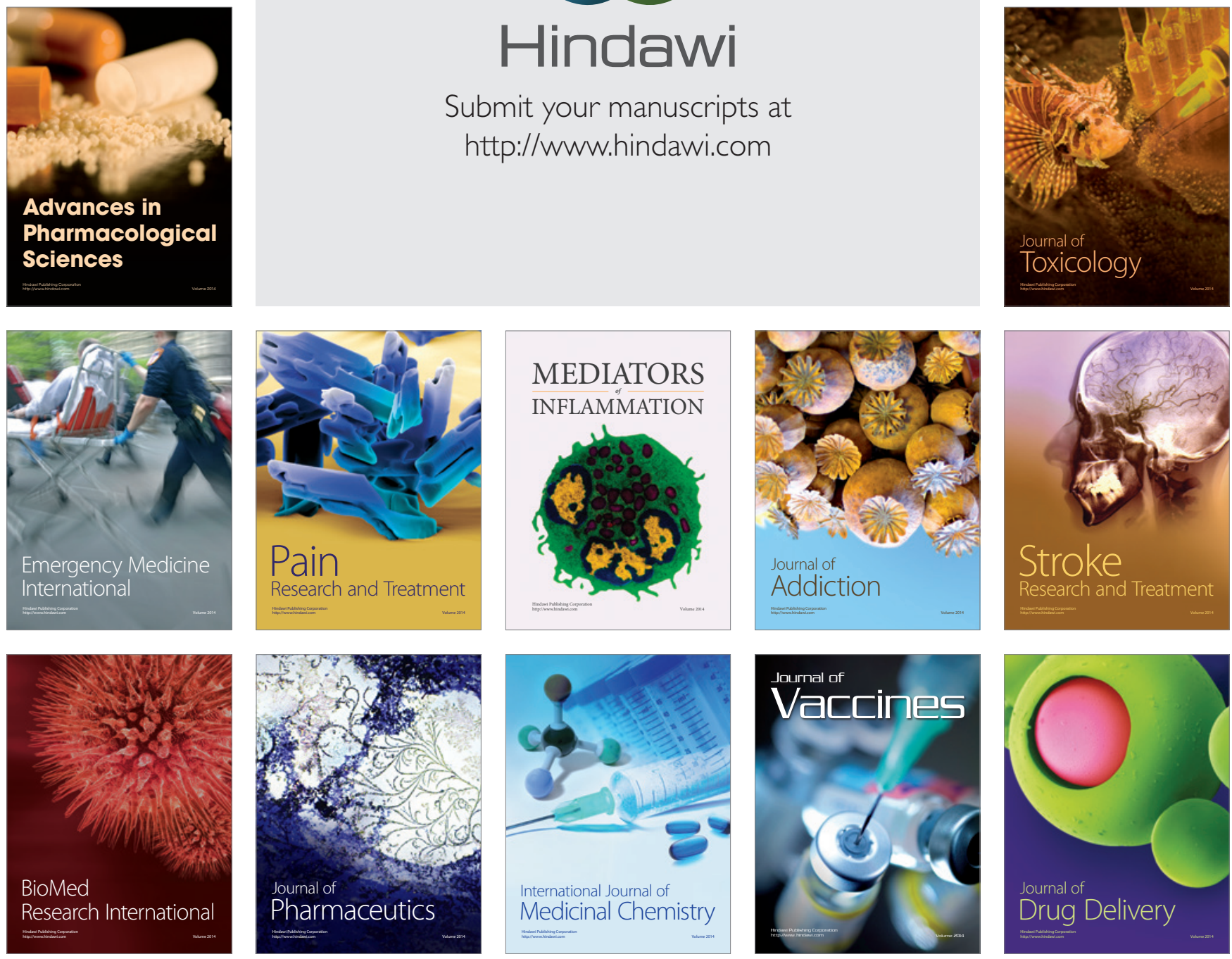